\title{
Epilepsiehäufigkeit wird durch ein Netz verschiedener Faktoren beeinflusst
}

Fragestellung: Wie hoch sind die Inzidenz und die Prävalenz von Epilepsie in verschiedenen Ländern, und welche Faktoren tragen dazu bei?

Hintergrund: Mittlerweile existieren viele Studien zur Prävalenz von Epilepsie aus verschiedenen Ländern. Die Prävalenzraten unterscheiden sich zum Teil deutlich. In dieser Metaanalyse wurden die Studien auf die Ursachen dieser Unterschiede hin untersucht.

Patienten und Methodik: In den Datenbanken MEDLINE und EMBASE wurden Studien bezüglich der Epilepsieprävalenz identifiziert und von zwei

Fiest KM, Sauro KM, Wiebe S et al. Prevalence and incidence of epilepsy: A systematic review and meta-analysis of international studies. Neurology 2017; 88: $296-303$ kommensstruktur und Alter der Patienten stratifiziert. Die Heterogenität der Studien wurde mittels ${ }^{2}$-Test evaluiert.

Ergebnisse: Von anfänglich 16.479 identifizierten Abstracts blieben nach dem Screening 48 Inzidenz- und 197-Prävalenzstudien (24 mit beiden Informationen) übrig. Die Punktprävalenz lag bei 6,38/1.000 (0,68\%), die Lebenszeitprävalenz bei 7,6/1.000. Aus Kamerun, Honduras und Panama wurden wesentlich höhere Raten berichtet. Die Epilepsieinzidenz lag bei $67,77 / 100.000$ (0,068\%). Die Rate war im Kindes- und Seniorenalter höher, die Studienanzahl ließ hier aber keine Metaanalyse zu. Die Prävalenz unterschied sich deutlich zwischen einkommensstarken $(0,049 \%)$ und -schwachen Ländern (0,139\%).

Schlussfolgerungen: Die Inzidenz- und Prävalenzraten liegen in etwa auf dem Niveau großer Einzelstudien. Die Schwankungsbreite mit teilweise zehnfach erhöhter Rate ließ sich nicht durch einen einzelnen Faktor, sondern durch die Kombination mehrerer Faktoren (Einkommen, ZNS-Infektionen, perinatales Risiko) erklären.

\section{- Kommentar von Jan Rémi, München}

\section{Inzidenz- und Prävalenzraten auf sehr solide Füße gestellt}

In dieser aufwändigen Metaanalyse werden die Inzidenz- und Prävalenzraten auf sehr solide Füße gestellt. Die typischerweise zitierte Prävalenzrate von 0,5-1\% bestätigt sich. Diese Studie kann gut als Basis für zukünftige epidemiologische Studien benutzt werden. Die durchschnittliche Prävalenzrate von $0,68 \%$ könnte nun als genauerer Wert statt der Spanne 0,5-1\% benutzt werden. Ein Einfluss des Niveaus der Gesundheitsversorgung wird aus der Studie sehr deutlich.

In Ländern mit weniger Zugang zu medizinischer Versorgung und anderen hygienischen Voraussetzungen wie zum Beispiel dem Zugang zu sauberem Wasser oder dem Auftre-

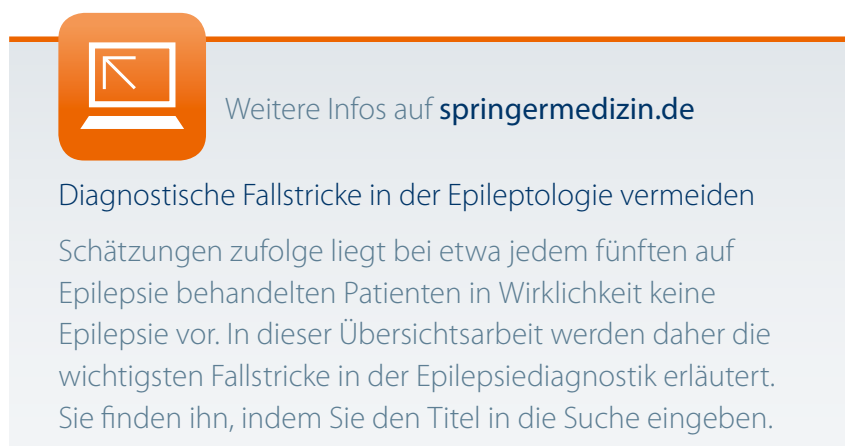

ten von Neurozystizerkose ist die Prävalenz höher. Hier können Vorsorge und frühe Behandlung hilfreich sein. Die Ergebnisse müssen insofern auch kritisch beleuchtet werden, als dass die Epilepsieraten in den Ländern mit hohem Einkommen oft keine populationsbasierten Daten darstellen, sondern sich häufig auf Daten aus der erstbehandelnden Einrichtung stützen. Somit könnte die Rate in diesen Ländern etwas unterschätzt sein. Dies sollte in zukünftigen Studien adressiert werden. Zudem ist der Bias der Unterberichtung durch Stigma und kulturelle Unterschiede in zu wenigen Studien untersucht. Epilepsie ist einer der häufigsten chronischen neurologischen Erkrankungen. Die Prävalenz liegt bei 0,68\% und die Einflussfaktoren für die Prävalenz sind soziökonomischer Status und Zugang zu Gesundheitsfürsorge und Hygiene.

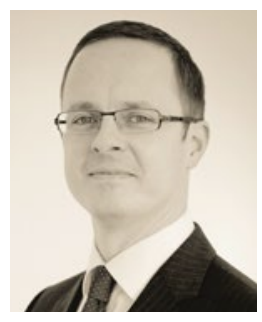

PD Dr. med. Jan Rémi, München

Neurologische Klinik und Poliklinik Klinikum der Universität München Großhadern

E-Mail: jan.remi@med.Imu.de 